Preprint typeset in JHEP style - PAPER VERSION

Cavendish-HEP-05/11

\title{
Distinguishing Spins in Supersymmetric and Universal Extra Dimension Models at the Large Hadron Collider*
}

\author{
Jennifer M. Smillie ${ }^{1}$ and Bryan R. Webber ${ }^{2}$ \\ Cavendish Laboratory, University of Cambridge \\ Madingley Road, Cambridge CB3 OHE, U.K. \\ ${ }^{1}$ E-mail: smillie@hep.phy.cam.ac.uk \\ ${ }^{2}$ E-mail: webber@hep.phy.cam.ac.uk
}

\begin{abstract}
An interesting alternative to supersymmetry (SUSY) for extending physics beyond the Standard Model is a model with universal extra dimensions (UED), in which the SUSY superpartners are replaced by Kaluza-Klein excitations of the Standard Model particles. If new particles are discovered at the LHC, even if their mass spectrum favours SUSY or UED, it will be vital to distinguish between their spin assignments in the two models as far as possible. We extend the method proposed by Barr [1] to the UED case and investigate the angular and charge asymmetries of decay distributions for sample mass spectra of both SUSY and UED types. For hierarchical ('SUSY-type') mass spectra there is a good chance of distinguishing the spin structures of the two models. However, a mass spectrum of the quasi-degenerate type expected in UED would make it difficult to observe spin correlations.
\end{abstract}

Keywords: Hadronic Colliders, Beyond Standard Model, Supersymmetry Phenomenology, Large Extra Dimensions.

*Work supported in part by the UK Particle Physics and Astronomy Research Council. 


\section{Contents}

1. Introduction 1

2. Decay chains in SUSY and UED 3

3. Spin correlations in SUSY 4

4. Spin correlations in UED 5

4.1 Correlations in $q^{*}$ and $Z^{*}$ decays 5

4.2 Quark + near lepton mass distribution 5

$\begin{array}{lll}4.3 & \text { Correlations in } l^{*} \text { decay } & 7\end{array}$

4.4 Dilepton mass distribution 8

4.5 Quark + far lepton mass distribution $\quad 8$

4.6 Observable quark-lepton correlations 9

5. Production cross sections 11

6. Experimental observables $\quad 13$

$\begin{array}{ll}\text { 7. Conclusions } & 15\end{array}$

A. Quark + far lepton correlation $\quad 16$

$\begin{array}{ll}\text { B. UED production cross sections } & 17\end{array}$

\section{Introduction}

The search for physics beyond the Standard Model (SM) will be a principal objective of the main experiments at the CERN Large Hadron Collider (LHC). The leading contender amongst theories of new physics is undoubtedly supersymmetry (SUSY), in which the SM particles have massive superpartners that differ from them by a half-unit of spin. The partners of strongly-interacting partons (the spartons) should be copiously produced once their production thresholds are passed. In R-parity conserving SUSY, the spartons typically decay into partons and electroweak sparticles, which themselves decay sequentially through the emission of SM particles to the stable lightest supersymmetric particle (LSP). The LSP is usually the lightest neutralino $\widetilde{\chi}_{1}^{0}$, which escapes from the detector unobserved. Thus the expected signature of SUSY is the copious production of high-energy jets and leptons plus large missing energy.

If the above signature of SUSY is observed at the LHC, it will be essential to confirm as far as possible that the produced objects are indeed superpartners and not some other 
manifestation of new physics. Methods are being developed for exploring the mass spectrum and decay systematics of candidate superparticles. ${ }^{1}$ The key issue then will be whether their spins fit the pattern expected for SUSY.

In this connection, a very interesting alternative hypothesis is that the new objects are indeed partners of the SM particles but with the same spins. This scenario is realized in the so-called universal extra dimension (UED) model, of the type proposed in refs. [4]. It is remarkable that UED and SUSY models could look very similar in collider experiments. In the former, the Kaluza-Klein (KK) excitations of the SM particles can carry a KK-parity analogous to the R-parity of the latter, implying pair production of the first KK-excitations and a stable lightest KK-particle (LKP), usually the first KK-excitation of the photon. If the energy of the LHC is insufficient to produce higher KK-excitations, then each SM particle would be found to have a single KK-partner with the same spin but a higher mass related to the size of the extra dimension.

It is not our intention here to advocate or criticise UED models as a viable alternative to SUSY, but simply to discuss the extent to which they could be distinguished from SUSY models with the same mass spectra and decay systematics. Admittedly certain mass spectra would already be suggestive of one model or the other: for example, the spectrum of KK-excitations is quasi-degenerate if the extra dimension is simply compactified on an $S_{1} / Z_{2}$ orbifold [5]. The degeneracy is broken only by zero-mode (SM) masses, volumesuppressed boundary terms and loop corrections with a relatively low cutoff. In SUSY models one usually assumes that soft SUSY-breaking terms satisfy universal boundary conditions at a much higher scale, so that such degeneracy at the weak scale would be unnatural. However, the number of unknowns and arbitrary assumptions in each case is so great that the exclusion of either class of model in favour of the other would only be truly convincing if their spin structures could be distinguished.

In the present paper we assume that a particular chain of decays that is common in both SUSY and UED models, starting from a squark or a KK-excited quark respectively, has been identified and that all the masses of the new particles involved in it are known. We then study the extent to which decay correlations, manifest in the invariant mass distributions of combinations of observable decay products, would enable one to distinguish between the SUSY and UED spin assignments of the new particles. Our work is thus an extension of that described in ref. [1] (see also [6]), where the SUSY decay correlations were compared with uncorrelated phase space. ${ }^{2}$

In the following section we present the decay chains to be considered, and in section 3 we recall the SUSY correlations investigated earlier. In section 4 we present our new results on the corresponding UED decay correlations. We derive simple analytical formulae for the correlation coefficients in terms of the masses in the decay chain, which should be of general use whatever the mass spectrum might turn out to be. We show graphical results for two specific mass scenarios, one considered more probable in SUSY and the other in UED. In both mass scenarios we compare the correlations predicted by the SUSY and UED spin assignments.

\footnotetext{
${ }^{1}$ See for example refs. [2, 3].

${ }^{2}$ Distinguishing between SUSY and UED at future $e^{+} e^{-}$colliders has been studied in refs. $[7,8]$.
} 
As was emphasised by Barr [1], the observability of interesting correlations depends crucially on the fact that the LHC is a proton-proton collider, so that squarks/KK-quarks are produced somewhat more copiously than their antiparticles. To quantify this effect, we need to know the direct and indirect production cross sections of KK-quarks and KKantiquarks. We have therefore computed the lowest-order two-parton to two-KK-parton matrix elements, which are expected to dominate the production of these particles. Our results, which differ somewhat from those presented in ref. $[9],{ }^{3}$ are discussed in section 5 and listed in appendix B.

Using our results on the UED production matrix elements and decay correlations, together with the decay branching ratios suggested in ref. [5], we have included a full simulation of the relevant UED processes in the HERWIG Monte Carlo event generator [10, 11]. Since the corresponding SUSY processes, with full spin correlations, are already a wellestablished feature of HERWIG [12,13], we are able in section 6 to present first detector-level results on distinguishing UED and SUSY spin correlations at the LHC. Our results and conclusions are summarized in section 7 .

\section{Decay chains in SUSY and UED}

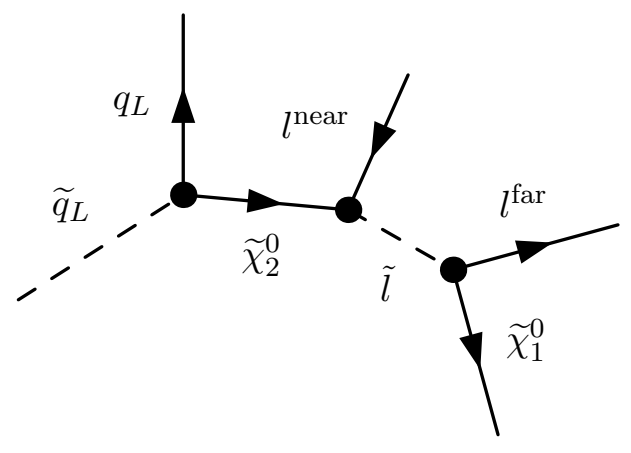

(a)

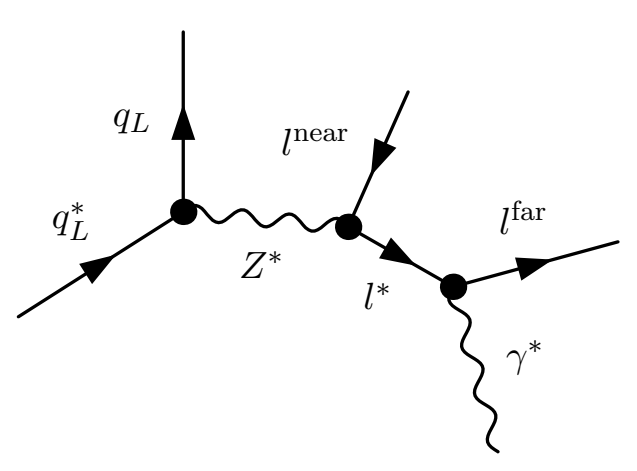

(b)

Figure 1: (a) SUSY and (b) UED decay chains considered here.

The SUSY decay chain that we shall consider, which is the same as that studied in ref. [1], is shown in figure 1, together with the corresponding UED process. In both cases the visible decay products are a quark jet and a pair of opposite-sign same-flavour (OSSF) leptons with the same chirality. We suppose that the new particle masses have been measured, either by an edge analysis along the lines of refs. [2, 3] or some other means, and it remains to decide whether the decay angular distributions agree better with the SUSY or UED spin assignments.

\footnotetext{
${ }^{3}$ An erratum to ref. [9] is in preparation (C. Macesanu, private communication).
} 
The angular distributions depend on whether or not the chirality of the slepton/KKlepton is the same at that of the decaying squark/KK-quark. ${ }^{4}$ For definiteness, we assume that the latter is left-handed, which is preferred in both of the models under consideration. We can then characterise the process by the chirality and charge of the "near" lepton, defined as shown in figure 1. Of course, we cannot distinguish experimentally between the "near" and "far" leptons, and so their contributions to any distribution will eventually have to be combined. However, in principle (in the zero-width approximation that we use) the processes with opposite "near" and "far" charge assignments are distinct. There are then two fundamental processes with different decay correlations, which (as in ref. [1]) we label 1 and 2:

- Process 1: $\left\{q, l^{\text {near }}, l^{\text {far }}\right\}=\left\{q_{L}, l_{L}^{-}, l_{L}^{+}\right\}$or $\left\{\bar{q}_{L}, l_{L}^{+}, l_{L}^{-}\right\}$or $\left\{q_{L}, l_{R}^{+}, l_{R}^{-}\right\}$or $\left\{\bar{q}_{L}, l_{R}^{-}, l_{R}^{+}\right\}$;

- Process 2: $\left\{q, l^{\text {near }}, l^{\text {far }}\right\}=\left\{q_{L}, l_{L}^{+}, l_{L}^{-}\right\}$or $\left\{\bar{q}_{L}, l_{L}^{-}, l_{L}^{+}\right\}$or $\left\{q_{L}, l_{R}^{-}, l_{R}^{+}\right\}$or $\left\{\bar{q}_{L}, l_{R}^{+}, l_{R}^{-}\right\}$.

\section{Spin correlations in SUSY}

We first recapitulate from ref. [1] the angular distributions that are expected in the SUSY decay chain $1(\mathrm{a})$. The $\widetilde{\chi}_{2}^{0}$ has spin one-half and its helicity is the same as that of the quark, since the squark is a scalar. Therefore a near lepton with the same helicity as the quark (process 1 ) will be emitted preferentially at large values of the angle $\theta^{*}$ between its direction and that of the quark in the $\widetilde{\chi}_{2}^{0}$ rest frame, with angular distribution (neglecting all SM particle masses)

$$
\frac{\mathrm{d} P_{1}^{\mathrm{SUSY}}}{\mathrm{d} \cos \theta^{*}}=\frac{1}{2}\left(1-\cos \theta^{*}\right)
$$

A near lepton with helicity opposite to the quark (process 2), on the other hand, will have angular distribution

$$
\frac{\mathrm{d} P_{2}^{\mathrm{SUSY}}}{\mathrm{d} \cos \theta^{*}}=\frac{1}{2}\left(1+\cos \theta^{*}\right)
$$

In terms of the $q l^{\text {near }}$ invariant mass,

$$
\left(m_{q l}^{\text {near }}\right)^{2}=2\left|\mathbf{p}_{l}\right|\left|\mathbf{p}_{q}\right|\left(1-\cos \theta^{*}\right)=\frac{1}{2}\left(m_{q l}^{\text {near }}\right)_{\max }^{2}\left(1-\cos \theta^{*}\right),
$$

defining the rescaled invariant mass variable to be

$$
\widehat{m}=m_{q l}^{\text {near }} /\left(m_{q l}^{\text {near }}\right)_{\max }=\sin \left(\theta^{*} / 2\right)
$$

we therefore have

$$
\frac{\mathrm{d} P_{1}^{\mathrm{SUSY}}}{\mathrm{d} \widehat{m}}=4 \widehat{m}^{3}
$$

and

$$
\frac{\mathrm{d} P_{2}^{\mathrm{SUSY}}}{\mathrm{d} \widehat{m}}=4 \widehat{m}\left(1-\widehat{m}^{2}\right)
$$

\footnotetext{
${ }^{4}$ We should emphasise that we use the term 'chirality' loosely here, since neither the sparticles nor the KK-excitations concerned have definite handedness: what we mean is that they couple to SM particles of that chirality.
} 
The slepton produced in the decay of the $\widetilde{\chi}_{2}^{0}$ is a scalar particle, and so its decay is isotropic in its rest frame, and the near and far lepton directions are uncorrelated in that frame. There is nevertheless a weak correlation between the directions of the far lepton and the quark, due to the relative velocity of the slepton and the $\widetilde{\chi}_{2}^{0}$, which we discuss in more detail in appendix A.

\section{Spin correlations in UED}

\subsection{Correlations in $q^{*}$ and $Z^{*}$ decays}

In the UED decay chain, the primary object has spin one-half and it decays to a quark and a vector boson, $q^{*} \rightarrow q Z^{*}$. The vector boson is either longitudinally or transversely polarised, with relative probabilities $m_{q^{*}}^{2}$ and $2 m_{Z^{*}}^{2}$, respectively. By angular momentum conservation, when the polarisation is transverse the helicity of the $Z^{*}$, and of the near lepton, must be the same as that of the quark. The distribution of the angle $\theta^{*}$ between the directions of the near lepton and the quark in the $Z^{*}$ rest frame is therefore of the form

$$
\frac{\mathrm{d} P_{1,2}^{\mathrm{UED}}}{\mathrm{d} \cos \theta^{*}}=\frac{1}{1+2 x}\left(\frac{\mathrm{d} P_{0}}{\mathrm{~d} \cos \theta^{*}}+2 x \frac{\mathrm{d} P_{\mp}}{\mathrm{d} \cos \theta^{*}}\right)
$$

where $x=m_{Z^{*}}^{2} / m_{q^{*}}^{2}$ and $P_{\lambda}$ on the right-hand side represents the distribution for $Z^{*}$ helicity $\lambda$.

In the rest frame of the $Z^{*}$, the decay of the longitudinally polarised state is forwardbackward symmetric, with angular distribution

$$
\frac{\mathrm{d} P_{0}}{\mathrm{~d} \cos \theta^{*}}=\frac{3}{2(2+y)}\left(\sin ^{2} \theta^{*}+y \cos ^{2} \theta^{*}\right)
$$

where $y=m_{l^{*}}^{2} / m_{Z^{*}}^{2}$. The transverse decay distribution for \pm ve helicity is

$$
\frac{\mathrm{d} P_{ \pm}}{\mathrm{d} \cos \theta^{*}}=\frac{3}{4(2+y)}\left[\left(1 \pm \cos \theta^{*}\right)^{2}+y \sin ^{2} \theta^{*}\right]
$$

The angular distributions for the two fundamental processes are therefore given by

$$
\frac{\mathrm{d} P_{1,2}^{\mathrm{UED}}}{\mathrm{d} \cos \theta^{*}}=\frac{3}{2(1+2 x)(2+y)}\left[1+x+x y \mp 2 x \cos \theta^{*}-(1-x)(1-y) \cos ^{2} \theta^{*}\right] .
$$

\subsection{Quark + near lepton mass distribution}

Using eq. (4.4), the distribution of the rescaled $q l^{\text {near }}$ invariant mass (3.4) is given in process 1 by

$$
\frac{\mathrm{d} P_{1}^{\mathrm{UED}}}{\mathrm{d} \widehat{m}}=\frac{6 \widehat{m}}{(1+2 x)(2+y)}\left[y+4(1-y+x y) \widehat{m}^{2}-4(1-x)(1-y) \widehat{m}^{4}\right]
$$

and in process 2 by

$$
\frac{\mathrm{d} P_{2}^{\mathrm{UED}}}{\mathrm{d} \widehat{m}}=\frac{6 \widehat{m}}{(1+2 x)(2+y)}\left[4 x+y+4(1-2 x-y+x y) \widehat{m}^{2}-4(1-x)(1-y) \widehat{m}^{4}\right] .
$$


Notice that the $\widehat{m}$ distributions for the two UED processes become identical as $x \rightarrow 0$, i.e. for $m_{Z^{*}} \ll m_{q^{*}}$, since in that limit the $Z^{*}$ polarisation is purely longitudinal. On the other hand when $x \rightarrow 1$ and $y \rightarrow 0\left(m_{l^{*}} \ll m_{Z^{*}} \sim m_{q^{*}}\right)$ the UED distributions become indistinguishable from those of the corresponding SUSY processes, eqs. (3.5,3.6).

The features of the $q l^{\text {near }}$ mass distributions can be illustrated by considering their forms for typical UED and SUSY patterns of new particle masses. A possible UED mass spectrum (from [5]) is given in table 1 for inverse radius $R^{-1}=500 \mathrm{GeV}$, cut-off $\Lambda$ such that $\Lambda R=20$ and $m_{h}=120 \mathrm{GeV}$. This model also assumes vanishing boundary terms at the cut-off scale $\Lambda$, and a vanishing boundary mass term for the Higgs mass, $\bar{m}_{h}^{2}$. The lightest four left KK-quarks are degenerate in mass and are labelled here collectively as $q_{L}^{*}$. Similarly the right and left KK-electrons and KK-muons are degenerate in mass and are labelled here as $l_{R}^{*}$ and $l_{L}^{*}$ respectively. This spectrum illustrates the feature of UED that the new particles have masses which are much closer to each other (usually within $100-200 \mathrm{GeV}$ ) than in a SUSY spectrum based on high-scale universality.

In the UED model we have eqs. (4.5) and (4.6) with $x=m_{Z^{*}}^{2} / m_{q^{*}}^{2}=0.803$; the $Z^{*}$ decays preferentially to a left-handed excited lepton and so we use $y=m_{l_{L}^{*}}^{2} / m_{Z^{*}}^{2}=0.923$, which yields

$$
\begin{aligned}
& \frac{\mathrm{d} P_{1}^{\mathrm{UED}}}{\mathrm{d} \widehat{m}}=0.727 \widehat{m}+2.577 \widehat{m}^{3}-0.047 \widehat{m}^{5}, \\
& \frac{\mathrm{d} P_{2}^{\mathrm{UED}}}{\mathrm{d} \widehat{m}}=3.257 \widehat{m}-2.483 \widehat{m}^{3}-0.047 \widehat{m}^{5} .
\end{aligned}
$$

\begin{tabular}{|c|c|c|c|c|}
\hline$\gamma^{*}$ & $Z^{*}$ & $q_{L}^{*}$ & $l_{R}^{*}$ & $l_{L}^{*}$ \\
\hline 501 & 536 & 598 & 505 & 515 \\
\hline
\end{tabular}

Table 1: UED masses in $\mathrm{GeV}$, for $R^{-1}=500 \mathrm{GeV}, \Lambda R=20, m_{h}=$ $120 \mathrm{GeV}, \bar{m}_{h}^{2}=0$ and vanishing boundary terms at cut-off scale $\Lambda$.

These should be compared with the corresponding SUSY expressions (3.5) and (3.6), which are independent of the particle masses.

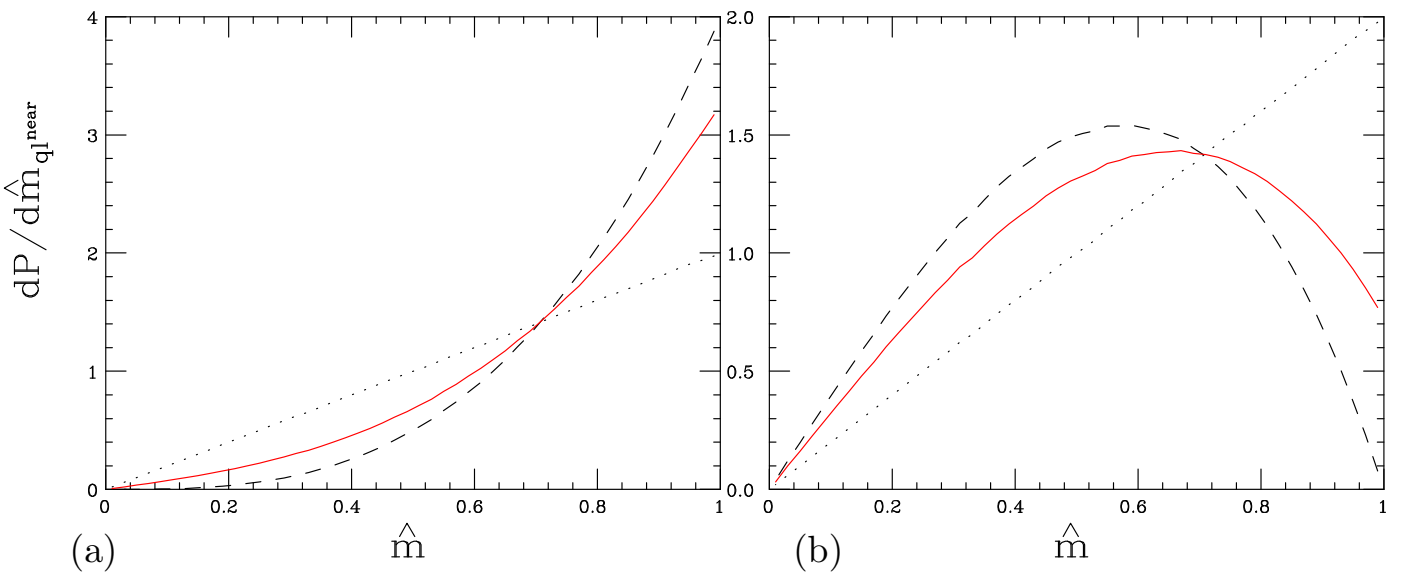

Figure 2: UED and SUSY distributions for (a) Process 1 and (b) Process 2 with respect to the rescaled $q l^{\text {near }}$ invariant mass, for the UED mass spectrum in table 1. Dotted: phase space. Dashed: SUSY. Solid/red: UED.

The UED and SUSY angular distributions are plotted against each other for processes 1 and 2 in figures $2(\mathrm{a})$ and $2(\mathrm{~b})$ respectively. Since $x=m_{Z^{*}}^{2} / m_{q^{*}}^{2}$ is large in the typical 
UED scenario, and the effect of $y=m_{l^{*}}^{2} / m_{Z^{*}}^{2}$ is weak at large $x$, the UED and SUSY distributions are similar. Therefore it will be difficult to verify the UED spin assignments if the spectrum is quasi-degenerate like that in table 1.

The SUSY mass spectrum, on the other hand, does not naturally have the same near-degeneracy of neutralinos and squarks, and therefore the UED and SUSY angular distributions are more distinct. For illustration, we consider the MSSM Snowmass point SPS 1a [14], which

\begin{tabular}{|c|c|c|c|c|}
\hline$\widetilde{\chi}_{1}^{0}$ & $\widetilde{\chi}_{2}^{0}$ & $\widetilde{u}_{L}$ & $\widetilde{e}_{R}$ & $\widetilde{e}_{L}$ \\
\hline 96 & 177 & 537 & 143 & 202 \\
\hline
\end{tabular}

Table 2: SUSY masses in GeV, for SPS point 1a. has the mass spectrum shown in table 2. The decay $\widetilde{\chi}_{2}^{0} \rightarrow l \tilde{l}_{R}$ is preferred and therefore we use $x=m_{\widetilde{\chi}_{2}^{0}}^{2} / m_{\widetilde{u}_{L}}^{2}=0.109$ and $y=m_{\widetilde{e}_{R}}^{2} / m_{\widetilde{\chi}_{2}^{0}}^{2}=$ 0.653 for the comparative UED distributions, giving

$$
\begin{aligned}
& \frac{\mathrm{d} P_{1}^{\mathrm{UED}}}{\mathrm{d} \widehat{m}}=1.213 \widehat{m}+3.108 \widehat{m}^{3}-2.301 \widehat{m}^{5}, \\
& \frac{\mathrm{d} P_{2}^{\mathrm{UED}}}{\mathrm{d} \widehat{m}}=2.020 \widehat{m}+1.493 \widehat{m}^{3}-2.301 \widehat{m}^{5} .
\end{aligned}
$$

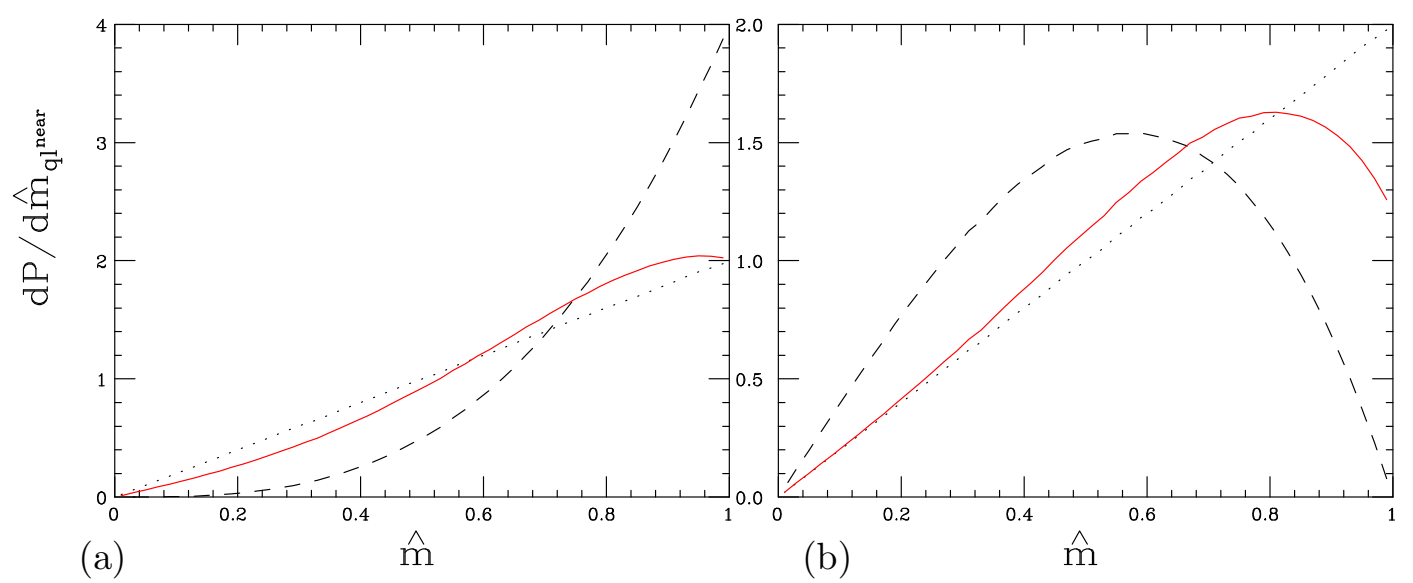

Figure 3: UED and SUSY distributions for (a) Process 1 and (b) Process 2 with respect to the rescaled $q l^{\text {near }}$ invariant mass, for the SUSY mass spectrum in table 2. Dotted: phase space. Dashed: SUSY. Solid/red: UED.

The resulting mass distributions are compared in fig. 3. Owing to the small value of $x$, the UED predictions for the two processes are similar to each other, and different from the SUSY predictions. This gives some grounds for optimism that, if the spectrum is hierarchical, like that in table 2, then the SUSY spin assignments can be confirmed or ruled out in comparison with the UED assignments.

\subsection{Correlations in $l^{*}$ decay}

In the SUSY decay chain (figure 1a), the slepton $\tilde{l}$ is spinless and therefore it decays isotropically in its rest frame. In the UED case (figure 1b), the spin of the KK lepton $l^{*}$ induces non-trivial correlations. Up to an overall constant, the full matrix elements for UED processes 1 and 2 , as defined in section 2, take the form

$$
|\overline{\mathcal{M}}|^{2} \propto 2 z(1-z) W_{l^{*}}+(1-2 z) W_{f}
$$


where $z=m_{\gamma^{*}}^{2} / m_{l^{*}}^{2}, f$ represents the far lepton and, for $l=l^{*}$ or $f$,

$$
\begin{aligned}
W_{l} & =(1-x)\left(2 p_{Z^{*}} \cdot p_{n} p_{Z^{*}} \cdot p_{l}+m_{Z^{*}}^{2} p_{n} \cdot p_{l}\right)-4 x\left(p_{n} \cdot p_{q} p_{Z^{*}} \cdot p_{l}+p_{n} \cdot p_{Z^{*}} p_{q} \cdot p_{l}\right)+ \\
& +\left\{\begin{array}{l}
8 x^{2} p_{n} \cdot p_{q} p_{q^{*}} \cdot p_{l} \text { for process } 1 \\
8 x^{2} p_{n} \cdot p_{q^{*}} p_{q} \cdot p_{l} \text { for process } 2
\end{array}\right.
\end{aligned}
$$

where, as before, $x=m_{Z^{*}}^{2} / m_{q^{*}}^{2}$, and $n$ represents the near lepton.

To specify the $l^{*}$ decay distribution, we define $\theta$ as the angle between the near and far leptons in the $l^{*}$ rest frame, and $\phi$ as the angle between the $q l^{\text {near }}$ and dilepton planes, in the same frame. Then we find

$$
\begin{aligned}
\frac{\mathrm{d}^{3} P_{1,2}^{\mathrm{UED}}}{\mathrm{d} \cos \theta^{*} \mathrm{~d} \cos \theta \mathrm{d} \phi}= & \frac{3}{4 \pi(1+2 x)(2+y)}\left[1+x+x y \mp 2 x \cos \theta^{*}-(1-x)(1-y) \cos ^{2} \theta^{*}\right. \\
& -\frac{1-2 z}{1+2 z}\left\{\left[1+x-x y \mp 2 x \cos \theta^{*}-(1-x)(1+y) \cos ^{2} \theta^{*}\right] \cos \theta\right. \\
& \left.\left.-2\left[(1-x) \cos \theta^{*} \pm x\right] \sqrt{y} \sin \theta^{*} \sin \theta \cos \phi\right\}\right] .
\end{aligned}
$$

\subsection{Dilepton mass distribution}

The dilepton mass $m_{l l}$ is simply related to the $l^{*}$ decay angle $\theta$ :

$$
m_{l l}^{2}=\frac{1}{4} x^{2}(1-y)(1-z)(1-\cos \theta) m_{q^{*}}^{2}
$$

and so, defining

$$
\widehat{m}_{l l}=m_{l l} /\left(m_{l l}\right)_{\max }=\sin (\theta / 2)
$$

we have

$$
\frac{\mathrm{d} P_{1,2}^{\mathrm{UED}}}{\mathrm{d} \widehat{m}_{l l}}=\frac{4 \widehat{m}_{l l}}{(2+y)(1+2 z)}\left[y+4 z+(2-y)(1-2 z) \widehat{m}_{l l}^{2}\right] .
$$

The dilepton mass distribution is potentially a good indicator of UED spin correlations, because it is the same for processes 1 and 2 and relatively easy to measure. We see from eq. (4.14) that the deviation from the linear mass spectrum of phase space or SUSY is greatest when $y$ and $z$ are small, i.e., when $m_{\gamma^{*}} \ll m_{l^{*}} \ll m_{Z^{*}}$. On the other hand, the spin correlation vanishes when $z=\frac{1}{2}$, that is, when $m_{\gamma^{*}}=m_{l^{*}} / \sqrt{2}$, which is close to the situation at SPS point 1a. The typical UED scenario with quasi-degenerate masses also yields a small effect, with a mass spectrum proportional to $\widehat{m}_{l l}\left(1-\widehat{m}_{l l}^{2} / 5\right)$. Therefore a dilepton mass distribution differing significantly from phase space or SUSY would only be manifest in a UED model substantially different from those considered so far.

\subsection{Quark + far lepton mass distribution}

The quark + far lepton mass is a function of all the decay angles,

$$
\begin{aligned}
\left(m_{q l}^{\mathrm{far}}\right)^{2}= & \frac{1}{4}(1-x)(1-z)\left[(1+y)\left(1-\cos \theta^{*} \cos \theta\right)+\right. \\
& \left.+(1-y)\left(\cos \theta^{*}-\cos \theta\right)-2 \sqrt{y} \sin \theta^{*} \sin \theta \cos \phi\right] m_{q^{*}}^{2}
\end{aligned}
$$




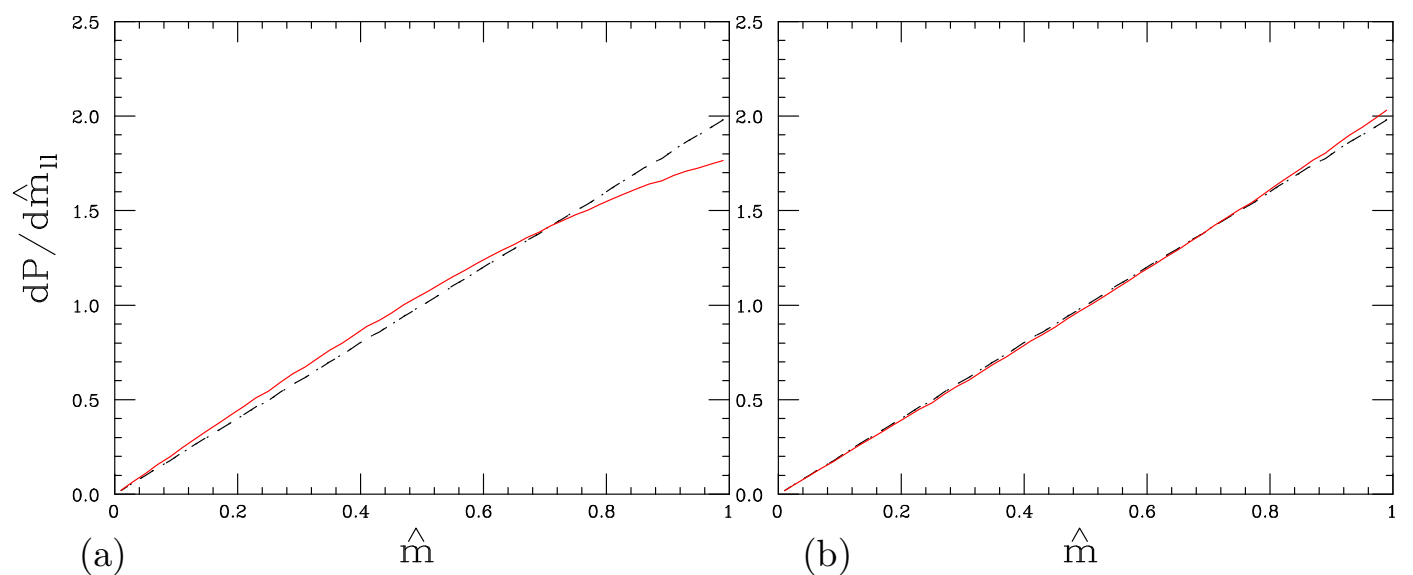

Figure 4: UED and SUSY distributions with respect to the rescaled dilepton invariant mass, for (a) the UED and (b) the SUSY mass spectrum given above. Dotted: phase space. Dashed: SUSY. Solid/red: UED.

The maximum value occurs for $\theta^{*}=0$ and $\theta=\pi$, when

$$
\left(m_{q l}^{\mathrm{far}}\right)_{\max }^{2}=(1-x)(1-z) m_{q^{*}}^{2},
$$

and therefore the rescaled quark + far lepton mass is given by

$$
\begin{aligned}
\widehat{m}_{q f} \equiv m_{q l}^{\mathrm{far}} /\left(m_{q l}^{\mathrm{far}}\right)_{\max }= & \frac{1}{2}\left[(1+y)\left(1-\cos \theta^{*} \cos \theta\right)+\right. \\
& \left.+(1-y)\left(\cos \theta^{*}-\cos \theta\right)-2 \sqrt{y} \sin \theta^{*} \sin \theta \cos \phi\right]^{\frac{1}{2}} .
\end{aligned}
$$

The phase space for this quantity increases linearly up to the point $\widehat{m}_{q f}=\sqrt{y}$, then decreases logarithmically to zero. In the region $\widehat{m}_{q f}<\sqrt{y}$, the probability distribution has a polynomial form, as illustrated in figures 5 and 6 for the UED and SUSY mass scenarios respectively. Equations for the distribution are given in appendix A.

We see that, as in the case of the dilepton mass distribution, the spin dependence of the quark + far lepton distribution is weak for both the mass spectra considered here.

\subsection{Observable quark-lepton correlations}

To proceed further, we must face the fact that the $q l^{\text {near }}$ and $q l^{\text {far }}$ mass distributions are not experimentally observable. As pointed out in ref. [1], the best that can be done in reality is to measure the invariant masses of jet + lepton combinations. Assuming that the jet and lepton are indeed decay products from process 1 or 2 , the $j l^{ \pm}$mass distribution for a given lepton charge receives near-lepton contributions from the corresponding process and the charge conjugate of the other process, plus far-lepton contributions from the other process and the charge conjugate of the same process. Concentrating on the UED scenario of a left-handed KK-quark decaying to a left-handed KK-lepton, we have

$$
\frac{\mathrm{d} P}{\mathrm{~d} m_{j l^{+}}}=f_{q}\left(\frac{\mathrm{d} P_{2}}{\mathrm{~d} m_{q l}^{\text {near }}}+\frac{\mathrm{d} P_{1}}{\mathrm{~d} m_{q l}^{\mathrm{far}}}\right)+f_{\bar{q}}\left(\frac{\mathrm{d} P_{1}}{\mathrm{~d} m_{q l}^{\text {near }}}+\frac{\mathrm{d} P_{2}}{\mathrm{~d} m_{q l}^{\mathrm{far}}}\right)
$$




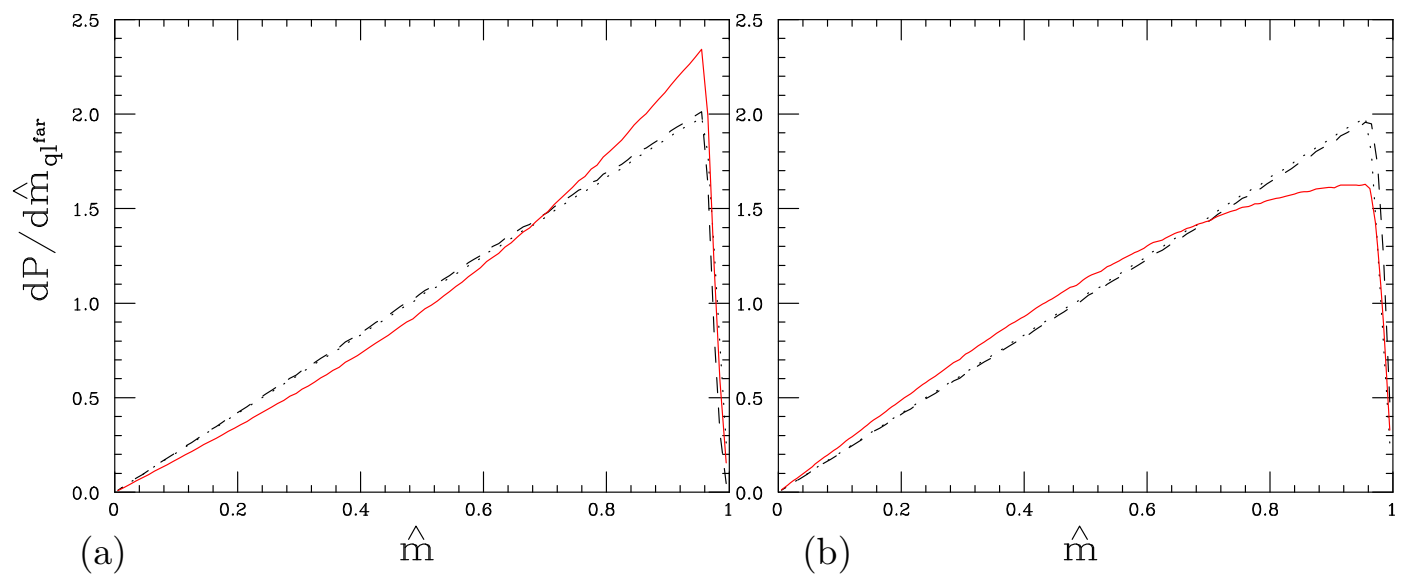

Figure 5: UED and SUSY distributions for (a) Process 1 and (b) Process 2 with respect to the rescaled $q l^{\text {far }}$ invariant mass, for the UED mass spectrum in table 1. Dotted: phase space. Dashed: SUSY. Solid/red: UED.

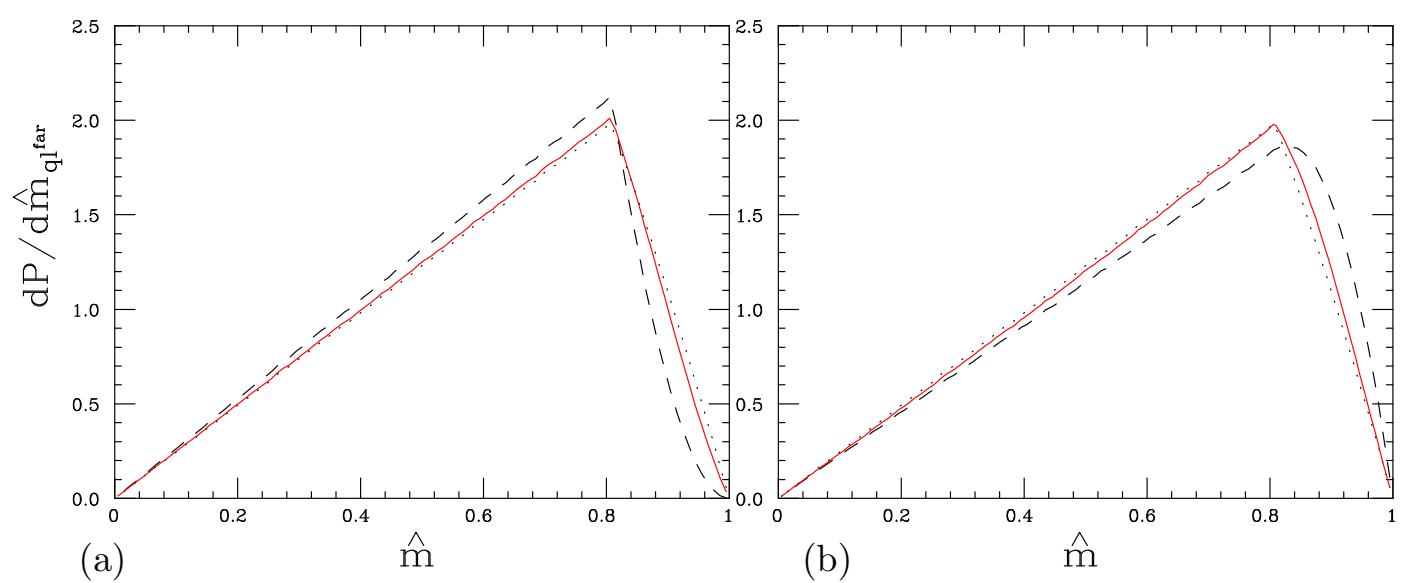

Figure 6: UED and SUSY distributions for (a) Process 1 and (b) Process 2 with respect to the rescsled $q l^{\text {far }}$ invariant mass, for the SUSY mass spectrum in table 2. Dotted: phase space. Dashed: SUSY. Solid/red: UED.

where $f_{q}$ and $f_{\bar{q}}$ are the quark and antiquark fractions in the selected event sample. Similarly

$$
\frac{\mathrm{d} P}{\mathrm{~d} m_{j l^{-}}}=f_{q}\left(\frac{\mathrm{d} P_{1}}{\mathrm{~d} m_{q l}^{\text {near }}}+\frac{\mathrm{d} P_{2}}{\mathrm{~d} m_{q l}^{\text {far }}}\right)+f_{\bar{q}}\left(\frac{\mathrm{d} P_{2}}{\mathrm{~d} m_{q l}^{\text {near }}}+\frac{\mathrm{d} P_{1}}{\mathrm{~d} m_{q l}^{\text {far }}}\right) .
$$

As will be discussed in section 5 , for both the UED and SUSY scenarios we find $f_{q} \simeq 0.7$, $f_{\bar{q}} \simeq 0.3$ at the LHC. The resulting observable jet+lepton mass distributions are then as depicted in figure 7 , where again we have normalised to the maximum observable mass.

Corresponding results for the SUSY mass scenario are shown in figure 8. Here the roles of processes 1 and 2 are interchanged in eqs. (4.18) and (4.19), since the decay now involves a right-handed slepton or KK-lepton. 


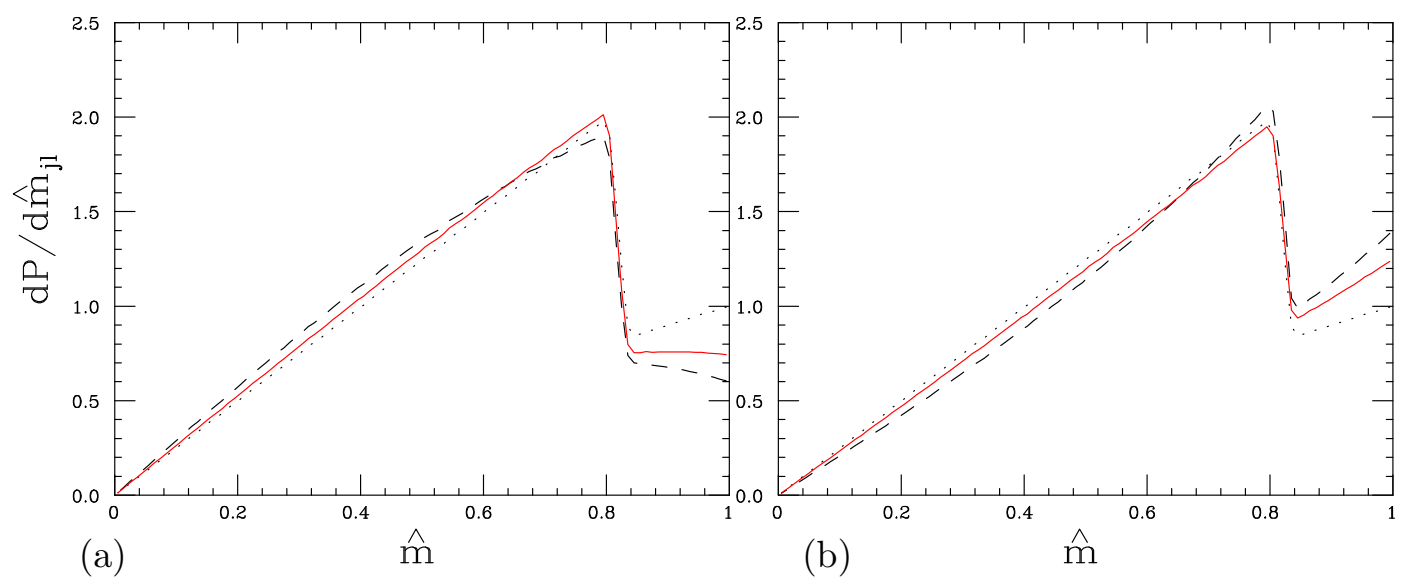

Figure 7: UED and SUSY rescaled mass distributions for (a) jet $+l^{+}$(b) jet $+l^{-}$, for the UED mass spectrum in table 1. Dotted: phase space. Dashed: SUSY. Solid/red: UED.

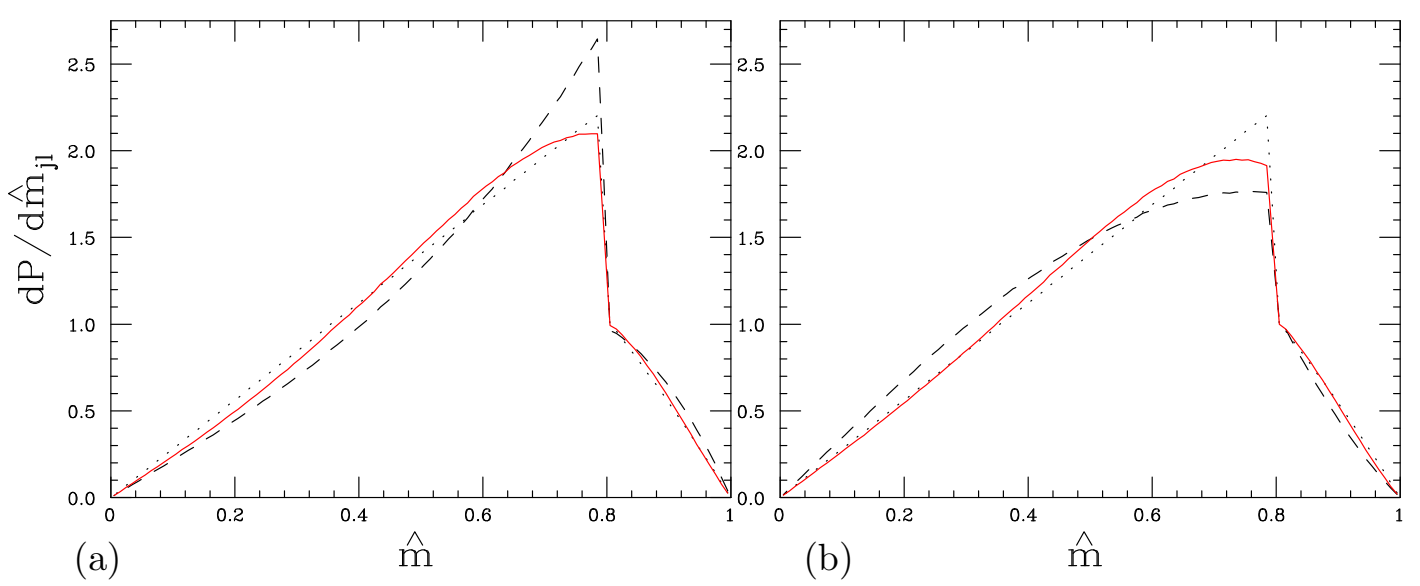

Figure 8: UED and SUSY rescaled mass distributions for (a) jet $+l^{+}$(b) jet $+l^{-}$, for the SUSY mass spectrum in table 2. Dotted: phase space. Dashed: SUSY. Solid/red: UED.

Figure 9 shows the resulting charge asymmetry

$$
A=\frac{\mathrm{d} P / \mathrm{d} m_{j l^{+}}-\mathrm{d} P / \mathrm{d} m_{j l^{-}}}{\mathrm{d} P / \mathrm{d} m_{j l^{+}}+\mathrm{d} P / \mathrm{d} m_{j l^{-}}}
$$

We see that the UED and SUSY charge asymmetries are similar in form, but the latter is smaller by a factor of $2-4$.

\section{Production cross sections}

As we saw in the previous section, the observability of a charge asymmetry sensitive to UED spin correlations depends critically on the difference between the production rates of KK-excited quarks and antiquarks, just as in SUSY it depends on the difference of squark and antisquark rates [1]. We have therefore computed the relevant lowest-order two-to-two scattering subprocess matrix elements and used them, together with the UED 


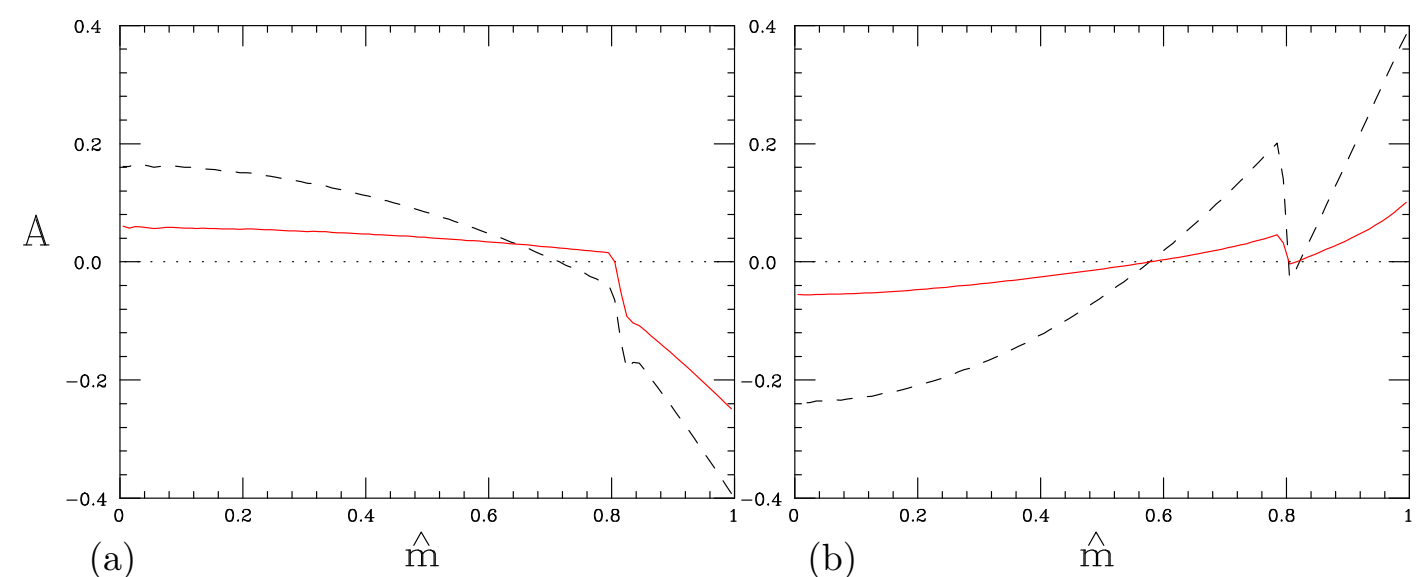

Figure 9: UED and SUSY charge asymmetries with respect to the jet + lepton rescaled invariant mass, for (a) the UED and (b) the SUSY mass spectrum given above. Dotted: phase space. Dashed: SUSY. Solid/red: UED.

branching ratios suggested in ref. [5], to estimate the UED production cross sections and the quantities $f_{q}$ and $f_{\bar{q}}$ appearing in eqs. (4.18) and (4.19).

Our expressions for the subprocess matrix elements are listed in appendix B. These results were obtained by including the Feynman rules for the effective four-dimensional theory in CompHEP [15]. They differ in several respects from those presented in ref. [9]. Details of the discrepancies are given in the appendix. Most importantly, we find a larger overall normalization.

Our numerical results for the production cross sections at the LHC are presented in table 3 . These results were obtained from parton-level Monte Carlo simulations of the production processes and decay chains, using the HERWIG event generator in SUSY mode with parton showering, hadronization and underlying event switched off. The HERWIG default (MRST leading-order [16]) parton distributions were used. For the UED simulations, the SUSY matrix element subroutine was replaced by a UED one and the SUSY particle data

\begin{tabular}{|c|c||c|c|c|c|}
\hline Masses & Model & $\sigma_{\text {all }}$ & $\sigma_{q^{*}}$ & $\sigma_{\bar{q}^{*}}$ & $f_{q}$ \\
\hline \hline UED & UED & 249 & 158 & 83 & 0.66 \\
UED & SUSY & 28 & 18 & 9 & 0.65 \\
SPS 1a & UED & 480 & 230 & 102 & 0.69 \\
SPS 1a & SUSY & 55 & 26 & 11 & 0.70 \\
\hline
\end{tabular}

Table 3: Production cross sections (pb) in UED and SUSY models, with UED or SUSY masses. input file consisted of UED data based on ref. [5].

As a result of the more singular structure of the matrix elements and the extra helicity states, the UED production cross sections tend to be larger than those of the analogous SUSY processes for identical mass spectra, leading to an overall enhancement of the cross section, by a factor of about 8 for both the mass scenarios that we studied. Thus a SUSYlike signature (e.g. many jets and leptons plus large missing energy) with a cross section 
an order of magnitude larger than that predicted by SUSY models could be an initial indication of UED.

The symbol $\sigma_{\text {all }}$ represents the sum of cross section for all two-to-two subprocesses included for that model, while $\sigma_{q^{*}}$ and $\sigma_{\bar{q}^{*}}$ represent the inclusive cross sections for direct and indirect production of the KK-quarks and -antiquarks (or squarks and antisquarks) initiating the decay chains in figure $1 .^{5}$ Although the overall magnitude of the cross sections is different in the UED and SUSY models, we see that the KK-quark or squark fraction $f_{q}$ remains at about $70 \%$ for both models in both mass scenarios.

\section{Experimental observables}

To investigate the observability of the effects discussed above, we switched on the parton showering, hadronization and underlying event in our HERWIG simulations and applied cuts to approximate those that might be used to select new physics experimentally. For jet cuts we used the simple calorimeter simulation and cone jet finder GETJET [17], with cone size $\Delta R=0.7$. Our cuts were as follows:

1. Missing transverse energy at least $100 \mathrm{GeV}$;

2. At least four jets with transverse energies $\left(E_{T}\right)$ above $50 \mathrm{GeV}$;

3. Sum of missing $E_{T}$ and four highest jet $E_{T}$ 's at least $400 \mathrm{GeV}$;

4. Quark jet plus lepton invariant masses within the allowed range: $m_{j l^{ \pm}} \leq\left(m_{q l}\right)_{\max }$.

For cut 4 we cheated somewhat by selecting the jet that is nearest (in $\Delta R$ ) to the true direction of the quark in the parton-level decay chain, and the leptons that do belong to that chain. Since we assume that all the new particle masses are already known, we expect that most ambiguities in the reconstruction of decay chains would in fact be resolved by invariant mass fits.

Of course more sophisticated cuts and detector simulations could be applied to suit particular experiments, but this analysis should at least give an indication of whether further efforts in that direction are warranted.

We included the UED spin correlations by generating decays according to phase space and reweighting events according to the invariant expression (4.9), evaluated at the parton level. To improve efficiency, we forced the decay $Z^{*} \rightarrow l^{*} l(l=e$ or $\mu)$, and correspondingly $\tilde{\chi}_{2}^{0} \rightarrow \tilde{l} l$, thereby enhancing the yield of the desired decay chains by factors of 3 and 8 in the UED and SUSY mass scenarios, respectively. To have comparable samples of each type, we generated $2 \times 10^{5}$ chains of each type in figure 1 (including their charge conjugates) for each mass scenario. However, because of the different production cross sections and branching ratios in UED and SUSY, this corresponds to varying effective luminosities, as summarised in table 4.

Also shown in table 4 are the fractions of events with the desired decay chains that survive the cuts listed above. For the SUSY mass scenario, the effect of these cuts is

\footnotetext{
${ }^{5}$ Top-flavoured KK-quarks or squarks were excluded as their decays would have a different signature.
} 


\begin{tabular}{|c|c||c|c|c|c|c|}
\hline Masses & Model & $\mathcal{L}_{\text {eff }}$ & Cut 1 & $1+2$ & $1+2+3$ & $1+2+3+4$ \\
\hline \hline UED & \multirow{2}{*}{ UED } & 7 & 0.52 & 0.11 & 0.11 & 0.05 \\
UED & \multirow{2}{*}{ SUSY } & 66 & 0.53 & 0.13 & 0.12 & 0.06 \\
SPS 1a & UED & 14 & 0.86 & 0.56 & 0.56 & 0.55 \\
SPS 1a & SUSY & 131 & 0.86 & 0.54 & 0.54 & 0.53 \\
\hline
\end{tabular}

Table 4: Effective luminosities $\left(\mathrm{fb}^{-1}\right)$ of our sample of $2 \times 10^{5}$ decay chains, and fractions surviving the cuts listed above.

not great. However, in the UED mass scenario the near-degeneracy of the mass spectrum means that the quark jet is likely to be relatively soft. It is therefore often misidentified or not found, with the result that few events survive the jet cuts.

Figures 10 and 11 show the reconstructed jet plus lepton mass distributions for the UED and SUSY mass scenarios, respectively. As we saw in section 4, the expected quasidegeneracy of the UED mass spectrum reduces the spin correlations and makes it hard to distinguish UED from SUSY in that case, even at the parton level. The experimental problems mentioned above, due to the relatively soft quark jet, further reduce the sensitivity and statistics. Consequently the charge asymmetry, shown in figure 12(a), is unlikely to be observable in the case of a UED-like mass spectrum.

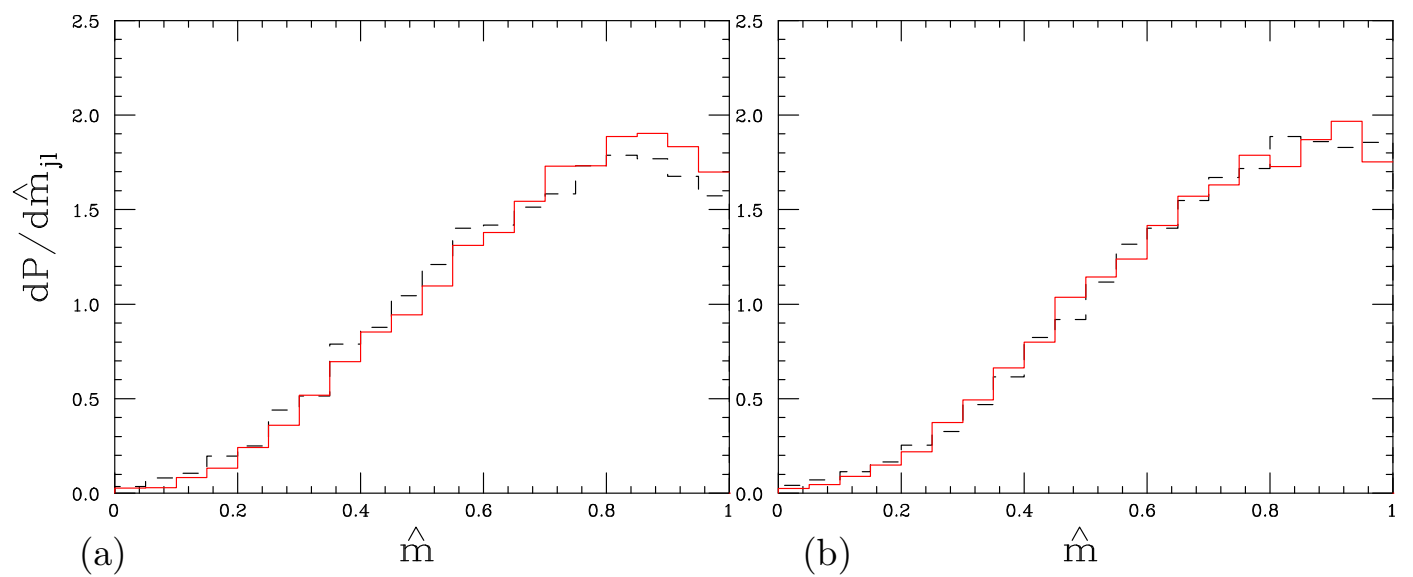

Figure 10: Detector-level rescaled mass distributions for (a) jet $+l^{+}$(b) jet $+l^{-}$, for the UED mass spectrum in table 1. Dashed: SUSY. Solid/red: UED.

The results for the SUSY (SPS 1a) mass spectrum, figures 11 and 12(b), are more encouraging. The spin correlations are larger in this case and, apart from some resolution smearing, their effects are not greatly diminished at the detector level. Correspondingly the charge asymmetry remains visible and similar to that predicted at the parton level, 


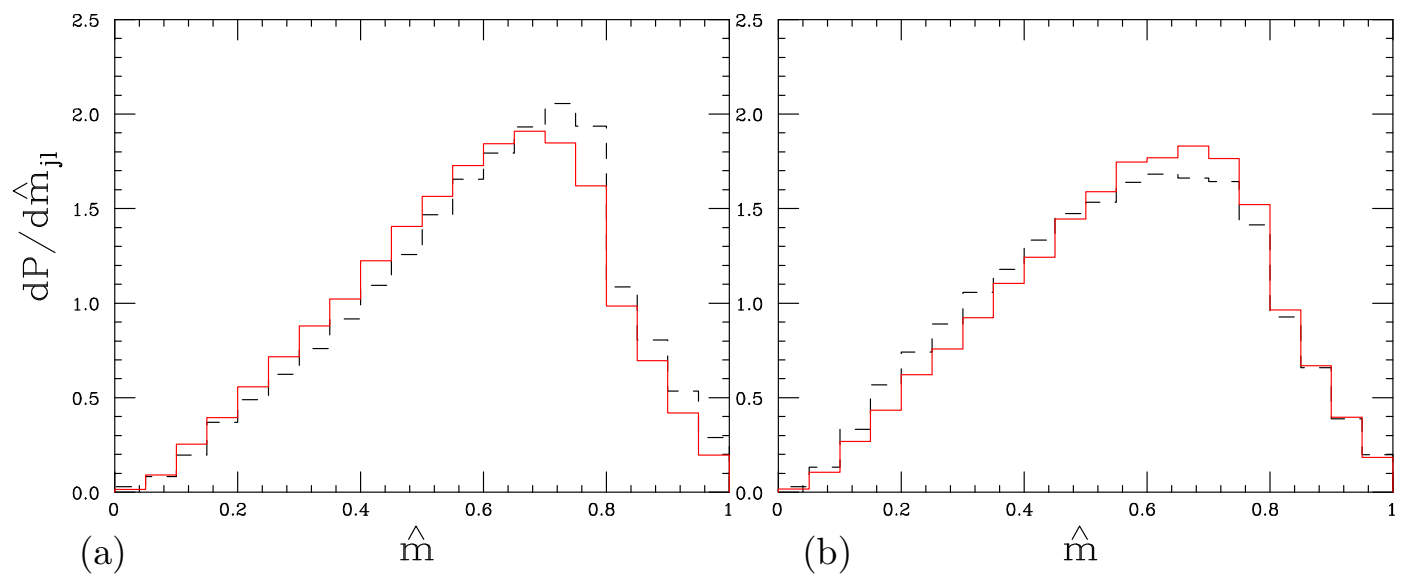

Figure 11: Detector-level rescaled mass distributions for (a) jet $+l^{+}$(b) jet $+l^{-}$, for the SUSY mass spectrum in table 2. Dashed: SUSY. Solid/red: UED.

except at very high and low masses, where the asymmetry is the ratio of two vanishing quantities.

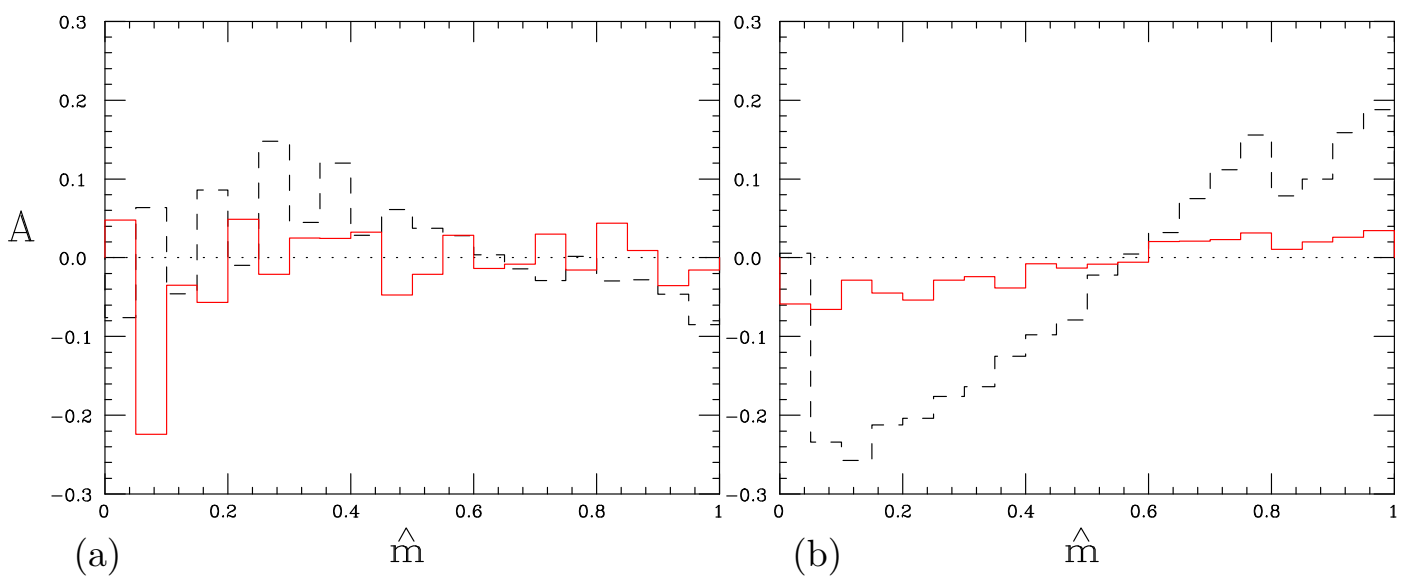

Figure 12: Detector-level charge asymmetries with respect to the jet + lepton rescaled invariant mass, for the (a) UED and (b) SUSY mass spectra given above. Dashed: SUSY. Solid/red: UED.

\section{Conclusions}

We have presented results of a comparative study of spin correlations in models with supersymmetry and universal extra dimensions. Complete results were obtained for a decay chain that is likely to be important if either model is relevant at LHC energies. The analytical expressions for two-particle invariant mass distributions in section 4 can be used to test the models for any combination of masses and chirality of the new particles involved in the decay chain. We presented numerical and graphical results for two particular mass scenarios: one UED-like and one SUSY-like (SPS 1a). In the former case the neardegeneracy of the mass spectrum of new particles would make it difficult to verify the spin 
content of the model in this way. In SUSY models such degeneracy would be less likely and the prospects for distinguishing between SUSY and UED spin assignments are better.

As an adjunct to our study of spin effects we rederived the production cross sections for KK-partons in UED models and found some differences from results in the literature. Due to the more singular matrix elements and extra helicity states, the cross sections are significantly larger than those for SUSY particles with the same mass spectrum. This could also serve as a means of discriminating between SUSY and UED.

\section{Acknowledgements}

We thank colleagues in the Cambridge SUSY Working Group for helpful discussions. We are especially grateful to Chris Lester for assistance in deriving the results on $q \mathrm{l}^{\text {far }}$ distributions in appendix A. We thank Cosmin Macesanu for valuable communications which led to the correction of errors in an earlier version of appendix B. BRW thanks Mihoko Nojiri for stimulating conversations and the Yukawa Institute, Kyoto University, and the CERN Theory Group for hospitality while part of this work was performed.

\section{Appendices}

\section{A. Quark + far lepton correlation}

The rescaled quark + far lepton invariant mass $\widehat{m}_{q f}$ is given in terms of the decay angles by eq. (4.17). The phase space distribution has the form

$$
\begin{aligned}
\frac{\mathrm{d} P^{\mathrm{PS}}}{\mathrm{d} \widehat{m}_{q f}} & =-2 \widehat{m}_{q f} \frac{\ln y}{1-y} & \text { for } 0 \leq \widehat{m}_{q f} \leq \sqrt{y} \\
& =-4 \widehat{m}_{q f} \frac{\ln \widehat{m}_{q f}}{1-y} & \text { for } \sqrt{y}<\widehat{m}_{q f} \leq 1 .
\end{aligned}
$$

In the SUSY decay chain the only non-trivial dependence is on the angle $\theta^{*}$. We find the following form for the rescaled mass distribution: for process 1

$$
\begin{aligned}
\frac{\mathrm{d} P_{1}^{\mathrm{SUSY}}}{\mathrm{d} \widehat{m}_{q f}} & =-\frac{4 \widehat{m}_{q f}}{(1-y)^{2}}(1-y+\ln y) & \text { for } 0 \leq \widehat{m}_{q f} \leq \sqrt{y} \\
& =-\frac{4 \widehat{m}_{q f}}{(1-y)^{2}}\left(1-\widehat{m}_{q f}^{2}+2 \ln \widehat{m}_{q f}\right) & \text { for } \sqrt{y}<\widehat{m}_{q f} \leq 1
\end{aligned}
$$

and for process 2

$$
\begin{aligned}
\frac{\mathrm{d} P_{2}^{\mathrm{SUSY}}}{\mathrm{d} \widehat{m}_{q f}} & =\frac{4 \widehat{m}_{q f}}{(1-y)^{2}}(1-y+y \ln y) & & \text { for } 0 \leq \widehat{m}_{q f} \leq \sqrt{y} \\
& =\frac{4 \widehat{m}_{q f}}{(1-y)^{2}}\left(1-\widehat{m}_{q f}^{2}+2 y \ln \widehat{m}_{q f}\right) & & \text { for } \sqrt{y}<\widehat{m}_{q f} \leq 1
\end{aligned}
$$


In the case of UED, there is non-trivial dependence on all the decay angles. We find the following form for the rescaled mass distribution: for process 1

$$
\begin{aligned}
\frac{\mathrm{d} P_{1}^{\mathrm{UED}}}{\mathrm{d} \widehat{m}_{q f}}= & \frac{12 \widehat{m}_{q f}}{(1+2 x)(2+y)(1+2 z)(1-y)^{2}}\{(1-y)[4 x-y+2(2+3 y-2 x(5+y)) z \\
& \left.-4 \widehat{m}_{q f}^{2}(2-3 x)(1-2 z)\right]-[y(1-2(4+y) z)+4 x(2 z-y(1-4 z)) \\
& \left.\left.+4 \widehat{m}_{q f}^{2}(1+y-x(2+y))(1-2 z)\right] \ln y\right\} \quad \text { for } 0 \leq \widehat{m}_{q f} \leq \sqrt{y} \\
= & \frac{12 \widehat{m}_{q f}}{(1+2 x)(2+y)(1+2 z)(1-y)^{2}}\left\{\left(1-\widehat{m}_{q f}^{2}\right)(4 x(1+2 y-5 z-6 y z)-5 y+2(2+9 y) z\right. \\
& \left.-4 \widehat{m}_{q f}^{2}(1-x)(1-z)\right]-2[y(1-2(4+y) z)+4 x(2 z-y(1-4 z)) \\
& \left.\left.+4 \widehat{m}_{q f}^{2}(1+y-x(2+y))(1-2 z)\right] \ln \widehat{m}_{q f}\right\} \quad \text { for } \sqrt{y}<\widehat{m}_{q f} \leq 1
\end{aligned}
$$

and for process 2

$$
\begin{aligned}
\frac{\mathrm{d} P_{2}^{\mathrm{UED}}}{\mathrm{d} \widehat{m}_{q f}}= & \frac{12 \widehat{m}_{q f}}{(1+2 x)(2+y)(1+2 z)(1-y)^{2}}\{(1-y)[-y+2(2+2 x(1-y)+3 y) z \\
& \left.-4 \widehat{m}_{q f}^{2}(2-x)(1-2 z)\right]-[y(1-2(4+y) z) \\
& \left.\left.+4 \widehat{m}_{q f}^{2}(1+(1-x) y)(1-2 z)\right] \ln y\right\} \quad \text { for } 0 \leq \widehat{m}_{q f} \leq \sqrt{y} \\
= & \frac{12 \widehat{m}_{q f}}{(1+2 x)(2+y)(1+2 z)(1-y)^{2}}\left\{\left(1-\widehat{m}_{q f}^{2}\right)[4(1+x) z-y(5-18 z+8 x z)\right. \\
& \left.-4 \widehat{m}_{q f}^{2}(1-x)(1-z)\right]-2[y(1-2(4+y) z) \\
& \left.\left.+4 \widehat{m}_{q f}^{2}(1+(1-x) y)(1-2 z)\right] \ln \widehat{m}_{q f}\right\} \quad \text { for } \sqrt{y}<\widehat{m}_{q f} \leq 1 .
\end{aligned}
$$

\section{B. UED production cross sections}

We neglect all Standard Model particle masses and work at tree level, so that the $n^{\text {th }}$ excited KK state of each particle has mass $n / R$. In practice we ignore $n>1$ excitations. We use here the notation of ref. [9]: $q_{1}^{\bullet}$ or $q_{1}^{\circ}$ represent the first KK-excitations of the $5 \mathrm{D}$ fields whose zero-modes are the left-handed doublet and right-handed singlet quarks respectively. Throughout, $q_{1}^{*}$ represents either $q_{1}^{\bullet}$ or $q_{1}^{\circ}, M_{1}=1 / R, s, t, u$ are the usual Mandelstam variables, $t_{3}=t-M_{1}^{2}$ and $u_{4}=u-M_{1}^{2}$. Only (B.1), (B.2) and (B.6) agree in form with ref. [9]. In addition we find an extra factor of 16 in the overall normalisation.

$$
\begin{gathered}
\bar{\sum}\left|\mathcal{M}\left(q \bar{q} \rightarrow q_{1}^{* \prime} \bar{q}_{1}^{* \prime}\right)\right|^{2}=\frac{4 g_{s}^{4}}{9}\left[\frac{2 M_{1}^{2}}{s}+\frac{t_{3}^{2}+u_{4}^{2}}{s^{2}}\right] \\
\bar{\sum}\left|\mathcal{M}\left(q \bar{q} \rightarrow q_{1}^{*} \bar{q}_{1}^{*}\right)\right|^{2}=\frac{g_{s}^{4}}{9}\left[2 M_{1}^{2}\left(\frac{4}{s}+\frac{s}{t_{3}^{2}}-\frac{1}{t_{3}}\right)\right. \\
\left.+\frac{23}{6}+\frac{2 s^{2}}{t_{3}^{2}}+\frac{8 s}{3 t_{3}}+\frac{6 t_{3}}{s}+\frac{8 t_{3}^{2}}{s^{2}}\right], \\
\bar{\sum}\left|\mathcal{M}\left(q q \rightarrow q_{1}^{*} q_{1}^{*}\right)\right|^{2}=\frac{g_{s}^{4}}{27}\left[M_{1}^{2}\left(6 \frac{t_{3}}{u_{4}^{2}}+6 \frac{u_{4}}{t_{3}^{2}}-\frac{s}{t_{3} u_{4}}\right)\right.
\end{gathered}
$$




$$
\begin{gathered}
\left.+2\left(3 \frac{t_{3}^{2}}{u_{4}^{2}}+3 \frac{u_{4}^{2}}{t_{3}^{2}}+4 \frac{s^{2}}{t_{3} u_{4}}-5\right)\right], \\
\bar{\sum}\left|\mathcal{M}\left(g g \rightarrow q_{1}^{*} \bar{q}_{1}^{*}\right)\right|^{2}=g_{s}^{4}\left[M_{1}^{4} \frac{-4}{t_{3} u_{4}}\left(\frac{s^{2}}{6 t_{3} u_{4}}-\frac{3}{8}\right)+M_{1}^{2} \frac{4}{s}\left(\frac{s^{2}}{6 t_{3} u_{4}}-\frac{3}{8}\right)\right. \\
\left.+\frac{s^{2}}{6 t_{3} u_{4}}-\frac{17}{24}+\frac{3 t_{3} u_{4}}{4 s^{2}}\right], \\
\bar{\sum}\left|\mathcal{M}\left(g q \rightarrow g^{*} q_{1}^{*}\right)\right|^{2}=\frac{-g_{s}^{4}}{3}\left[\frac{5 s^{2}}{12 t_{3}^{2}}+\frac{s^{3}}{t_{3}^{2} u_{4}}+\frac{11 s u_{4}}{6 t_{3}^{2}}+\frac{5 u_{4}^{2}}{12 t_{3}^{2}}+\frac{u_{4}^{3}}{s t_{3}^{2}}\right], \\
\bar{\sum}\left|\mathcal{M}\left(q \bar{q}^{\prime} \rightarrow q_{1}^{*} \bar{q}_{1}^{* \prime}\right)\right|^{2}=\frac{g_{s}^{4}}{18}\left[4 M_{1}^{2} \frac{s}{t_{3}^{2}}+5+4 \frac{s^{2}}{t_{3}^{2}}+8 \frac{s}{t_{3}}\right], \\
\bar{\sum}\left|\mathcal{M}\left(q q^{\prime} \rightarrow q_{1}^{*} q_{1}^{* \prime}\right)\right|^{2}=\frac{2 g_{s}^{4}}{9}\left[-M_{1}^{2} \frac{s}{t_{3}^{2}}+\frac{1}{4}+\frac{s^{2}}{t_{3}^{2}}\right] \\
\left.\bar{\sum} q_{1}^{\bullet} q_{1}^{\circ}\right)\left.\right|^{2}=\frac{g_{s}^{4}}{9}\left[M_{1}^{2}\left(\frac{2 s^{3}}{t_{3}^{2} u_{4}^{2}}-\frac{4 s}{t_{3} u_{4}}\right)+2 \frac{s^{4}}{t_{3}^{2} u_{4}^{2}}-8 \frac{s^{2}}{t_{3} u_{4}}+5\right], \\
\bar{\sum}\left|\mathcal{M}\left(q \bar{q}^{\prime} \rightarrow q_{1}^{\bullet} \bar{q}_{1}^{\prime \circ}\right)\right|^{2}=\frac{g_{s}^{4}}{9}\left[2 M_{1}^{2}\left(\frac{1}{t_{3}}+\frac{u_{4}}{t_{3}^{2}}\right)+\frac{5}{2}+\frac{4 u_{4}}{t_{3}}+\frac{2 u_{4}^{2}}{t_{3}^{2}}\right],
\end{gathered}
$$

which is the same result as for $q \bar{q} \rightarrow q_{1}^{\bullet} \bar{q}_{1}^{\circ}, q_{1}^{\circ} \bar{q}_{1}^{\bullet}$ and $q \bar{q}^{\prime} \rightarrow q_{1}^{\circ} \bar{q}_{1}^{\bullet \bullet}$ as these are given by the same $t$-channel diagram.

$$
\bar{\sum}\left|\mathcal{M}\left(q q^{\prime} \rightarrow q_{1}^{\bullet} q_{1}^{\prime \circ}\right)\right|^{2}=\frac{g_{s}^{4}}{9}\left[-2 M_{1}^{2}\left(\frac{1}{t_{3}}+\frac{u_{4}}{t_{3}^{2}}\right)+\frac{1}{2}+\frac{2 u_{4}^{2}}{t_{3}^{2}}\right]
$$

which is the same result as for $q q^{\prime} \rightarrow q_{1}^{\circ} q_{1}^{\prime \bullet}{ }^{6}$

$$
\begin{aligned}
& \bar{\sum}\left|\mathcal{M}\left(g g \rightarrow g^{*} g^{*}\right)\right|^{2}=\frac{9 g_{s}^{4}}{4}\left[3 M_{1}^{4} \frac{s^{2}+t_{3}^{2}+u_{4}^{2}}{t_{3}^{2} u_{4}^{2}}-3 M_{1}^{2} \frac{s^{2}+t_{3}^{2}+u_{4}^{2}}{s t_{3} u_{4}}\right. \\
& \left.+1+\frac{\left(s^{2}+t_{3}^{2}+u_{4}^{2}\right)^{3}}{4 s^{2} t_{3}^{2} u_{4}^{2}}-\frac{t_{3} u_{4}}{s^{2}}\right] \text {, } \\
& \bar{\sum}\left|\mathcal{M}\left(q \bar{q} \rightarrow g^{*} g^{*}\right)\right|^{2}=\frac{2 g_{s}^{4}}{27}\left[M_{1}^{2}\left(-\frac{4 s^{3}}{t_{3}^{2} u_{4}^{2}}+\frac{57 s}{t_{3} u_{4}}-\frac{108}{s}\right)\right. \\
& \left.+\frac{20 s^{2}}{t_{3} u_{4}}-93+\frac{108 t_{3} u_{4}}{s^{2}}\right] \text {. }
\end{aligned}
$$

\footnotetext{
${ }^{6}$ We are grateful to Martyn Gigg and Peter Richardson for pointing out that the result for these processes had mistakenly been taken to be the same as equation (B.9) in an earlier version.
} 


\section{References}

[1] A. J. Barr, Determining the spin of supersymmetric particles at the LHC using lepton charge asymmetry, Phys. Lett. B 596 (2004) 205 [arXiv:hep-ph/0405052].

[2] B. C. Allanach, C. G. Lester, M. A. Parker and B. R. Webber, Measuring sparticle masses in non-universal string inspired models at the LHC, JHEP 0009 (2000) 004 [arXiv:hep-ph/0007009].

[3] C. G. Lester, Model independent sparticle mass measurements at ATLAS, CERN-THESIS-2004-003

[4] T. Appelquist, H. C. Cheng and B. A. Dobrescu, Bounds on universal extra dimensions, Phys. Rev. D 64 (2001) 035002 [arXiv:hep-ph/0012100].

[5] H. C. Cheng, K. T. Matchev and M. Schmaltz, Radiative corrections to Kaluza-Klein masses, Phys. Rev. D 66 (2002) 036005 [arXiv:hep-ph/0204342]; Bosonic supersymmetry? Getting fooled at the LHC, ibid. 66 (() 2002) 056006 [arXiv:hep-ph/0205314].

[6] T. Goto, K. Kawagoe and M. M. Nojiri, Study of the slepton non-universality at the CERN Large Hadron Collider, Phys. Rev. D 70, 075016 (2004) [Erratum-ibid. D 71, 059902 (2005)] [arXiv:hep-ph/0406317].

[7] G. Bhattacharyya, P. Dey, A. Kundu and A. Raychaudhuri, Probing universal extra dimension at the International Linear Collider, arXiv:hep-ph/0502031.

[8] M. Battaglia, A. Datta, A. De Roeck, K. Kong and K. T. Matchev, Contrasting supersymmetry and universal extra dimensions at the CLIC multi-TeV e+ e-collider, arXiv:hep-ph/0502041.

[9] C. Macesanu, C. D. McMullen and S. Nandi, Collider implications of universal extra dimensions, Phys. Rev. D 66 (2002) 015009 [arXiv:hep-ph/0201300].

[10] G. Corcella, I.G. Knowles, G. Marchesini, S. Moretti, K. Odagiri, P. Richardson, M. H. Seymour and B. R. Webber, HERWIG 6: An event generator for hadron emission reactions with interfering gluons (including supersymmetric processes), JHEP 0101 (2001) 010 [arXiv:hep-ph/0011363].

[11] G. Corcella et al., HERWIG 6.5 release note, arXiv:hep-ph/0210213.

[12] P. Richardson, Spin correlations in Monte Carlo simulations, JHEP 0111 (2001) 029 [arXiv:hep-ph/0110108].

[13] S. Moretti, K. Odagiri, P. Richardson, M. H. Seymour and B. R. Webber, Implementation of supersymmetric processes in the HERWIG event generator, JHEP 0204, 028 (2002) [arXiv:hep-ph/0204123].

[14] B. C. Allanach et al., The Snowmass points and slopes: Benchmarks for SUSY searches, in Proc. of the APS/DPF/DPB Summer Study on the Future of Particle Physics (Snowmass 2001) ed. N. Graf, Eur. Phys. J. C 25 (2002) 113 [eConf C010630 (2001) P125] [arXiv:hep-ph/0202233].

[15] A. Pukhov et al., CompHEP: A package for evaluation of Feynman diagrams and integration over multi-particle phase space. User's manual for version 33, arXiv:hep-ph/9908288.

[16] A. D. Martin, R. G. Roberts, W. J. Stirling and R. S. Thorne, Scheme dependence, leading order and higher twist studies of MRST partons, Phys. Lett. B 443 (1998) 301

[arXiv:hep-ph/9808371]. 
[17] F. Paige, private communication. 